\title{
Unsteady MHD Free Convective Flow Past an Inclined Parabolic Accelerated Plate with Hall Current, Radiation Effects and Variable Temperature in a Porous Medium
}

\author{
Gilbert Magiboi Nalisi ${ }^{1}$, Jeconia Abongo Okello ${ }^{1}$, Johana Kibet Sigey ${ }^{1}$, \\ Mark Erick. Mwiti Kimathi ${ }^{2}$ \\ ${ }^{1}$ Department of Pure and Applied Mathematics, Jomo Kenyatta University of Agriculture and Technology, Nairobi, Kenya \\ ${ }^{2}$ Department of Industrial and Engineering Mathematics, Technical University of Kenya, Nairobi, Kenya \\ Email address: \\ nalisi2015@gmail.com (G. M. Nalisi),masenoj@gmail.com (J. A. Okello),jksigey@jkuat.ac.ke (J. K. Sigey), \\ memkimathi@gmail.com (M. E. M. Kimathi)
}

\section{To cite this article:}

Gilbert Magiboi Nalisi, Jeconia Abongo Okello, Johana Kibet Sigey, Mark Erick. Mwiti Kimathi. Unsteady MHD Free Convective Flow Past an Inclined Parabolic Accelerated Plate with Hall Current, Radiation Effects and Variable Temperature in a Porous Medium. International Journal of Management and Fuzzy Systems. Vol. 3, No. 2, 2017, pp. 15-27. doi: 10.11648/j.ijmfs.20170302.11

Received: November 4, 2016; Accepted: March 24, 2017; Published: April 25, 2017

\begin{abstract}
We analyze the effects of hall current and radiation on unsteady hydromagnetic free convection flow of a viscous incompressible electrically conducting fluid past an inclined parabolic accelerated plate in a porous medium by applying a transverse magnetic field which makes an angle $\alpha$ to the inclined plate. The fluid is assumed to be viscous, incompressible and electrically conducting with a strong magnetic field. Using the modified Ohm's law and the Bossinesq approximation the governing equations of the problem are reduced to local non-similarity boundary layer equations using suitable transformation.The dimensionless governing equations of flow field are solved numerically by Crank-Nicolson finite difference method for different values of governing flow parameters. The velocity and temperature profiles are shown through graphs.
\end{abstract}

Keywords: MHD Free Convection Flows, Heat Transfer, Hall Currents, Radiation, Transverse Magnetic Field, Inclined Parabolic Accelerated Plate, Porous Medium

\section{Introduction}

A current carrying conductor in a magnetic field experiences a force that tends to move it perpendicularly to the field. In this case of an electrically conducting parabolic accelerated fluid at low pressure, there is an interaction of the magnetic field with the electric field of both the electrons and the ionized atoms of the fluid. If the magnetic field is perpendicular to the electric field a current is induced in the conductive accelerated fluid whose direction is perpendicular to both the electric field and the magnetic field. This current is called Hall current and is induced by a phenomenon known as Hall Effect.Extensive reviews of theory of MHD flows have been done by many researchers. Kirimi. J. et al (2012) studied Magnetic field and Hall current effect on MHD free convective flow past a vertical rotating plate.Sugunammaet al (2013) considered inclined magnetic field and chemical reaction effects on flow over a semi infinite vertical porous plate through porous media. Sarkar B.C (2013) considered Hall effects on unsteady MHD free convective flow past an accelerated moving vertical plate with viscous dissipations. Murali. G. et al (2013) considered unsteady Magnetohydrodynamic free convective flow past a vertical plate. Sigey. K. J. (2013) studied Magnetohydrodynamic (MHD) free convective flow past an infinite vertical porous plate with joule heating. Amenya R.O. et al (2013) studied MHD free convection flow past a vertical infinite porous plate in the presence of transverse magnetic field with constant heat flux.Amos. S.I et al (2014) considered Numerical solution for thermal radiation effect on inclined magnetic field of MHD free convective heat transfer dissipative fluid flow past a moving vertical porous plate with variable suction.Idowu et al (2014) studied Numerical solution for thermal radiation effect on inclined magnetic field of MHD free convective Heat transfer dissipative fluid 
flow past a moving vertical porous plate with variable suction.Armstrong A.N. and Muthucumaraswamy R. (2014) considered MHD flow past a parabolic started vertical plate with variable temperature and mass diffusion. Muthucumaraswamy. R and Geetha. E. (2014) considered the Effects of parabolic motion on isothermal vertical plate with constant mass flux. Pattnaik. J.R. et al (2015) considered Radiation and mass transfer effects on MHD flow through porous medium past an exponentially accelerated plate with variable temperature. Kumar. J.G and Ramana R. M. (2015) considered viscous dissipation effects on MHD flow past a parabolic started plate with variable temperature and mass diffusion.Sharma G.K (2016) et al studied Unsteady Flow through Porous Media Past on Moving Vertical Plate with Variable Temperature in the Presence of Inclined Magnetic Field. Gaurav K.S et al (2016) considered unsteady flow through porous media past a moving vertical plate with variable temperature in the presence of inclined magnetic field. In this paper we consider hall current and radiation effects, inclined parabolic accelerated plate, variable temperature, a transverse magnetic field which makes an angle of $\alpha$ with the inclined plate and the vertical.

\section{Mathematical Model}

i) Flow is unsteady and laminar.

ii) The coordinate system is chosen in such a way that $x^{+}-$axis is considered along the porous plate; $y^{+}-$ axis is taken along the width of the plate and $z^{+}-$ axis normal to the plane of the plate.

iii) The fluid under consideration is Newtonian, viscous, incompressible and electrically conducting with constant physical properties.

iv) The magnetic Reynolds number is assumed to be small enough so that the induced magnetic field is negligible.

v) The effect of viscous dissipation is neglected in the energy equation.

vi) It is assumed that there is no applied voltage, which implies the absence of an electric field.

vii) Soret and Dufour effects are neglected.

viii) We employ a Darcian viscous model for porous medium.

ix) The velocity vector is of the form $\vec{q}=\left(u^{+}, v^{+}, 0\right)$

$\mathrm{x}$ ) A strong a transverse magnetic field which makes an angle of $\alpha$ with the inclined plate and the vertical.

xi) There is no chemical reaction between the diffusing species and the fluid.

xii) Liquid metals and ionized gases have permeability $\mu_{e}$ so that we write $\mathrm{B}=\mu_{e} \mathrm{H}$ in the frame of references.

xiii) All the physical properties of the fluid are considered to be constant.

xiv) All velocities are small compared with that of light $q^{2} / C^{2} \ll 1$

xv) Thermal conductivity $\kappa$ is assumed constant.

Under all this assumption the physical configuration and coordinate system of this study is presented in the figure below

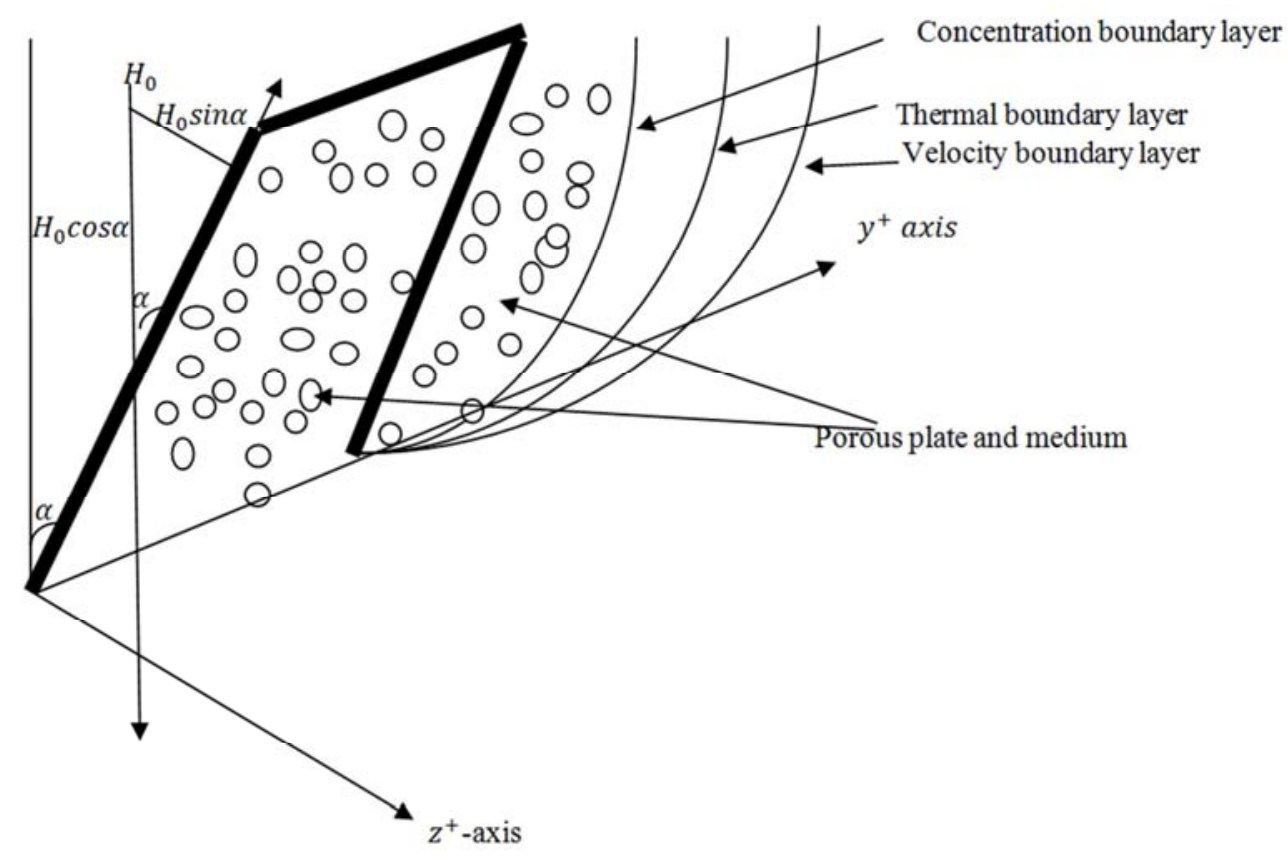

Figure 1. Flow configuration.

Taking into consideration the assumptions made above, the governing equations for natural convection flow with heat transfer of an electrically conducting, viscous, incompressible, optically thin radiating fluid through a porous medium taking Hall current into account, under
Boussinesq approximation are given by:

\subsection{Continuity Equation}

$$
\frac{\partial w^{+}}{\partial z^{+}}=0
$$




\subsection{Momentum Equation}

$$
\begin{gathered}
\left(\frac{\partial u^{+}}{\partial t^{+}}-w_{0}^{+} \frac{\partial u^{+}}{\partial z^{+}}\right)=v \frac{\partial^{2} u^{+}}{\partial z^{+2}}+g \beta_{T}\left(T^{+}-T_{\alpha}^{+}\right) \cos \alpha+ \\
\frac{\sigma \mu_{\mathrm{e}}^{2} H_{0}^{2} \sin ^{2} \alpha}{\rho} \frac{\left(m \mathrm{v}^{+} \sin \alpha-u^{+}\right)}{\left(1+m^{2} \sin ^{2} \alpha\right)}-u^{+} \frac{v}{K^{+}} \ldots . . \\
\left(\frac{\partial \mathrm{v}^{+}}{\partial \mathrm{t}^{+}}-\mathrm{w}_{0}^{+} \frac{\partial \mathrm{v}^{+}}{\partial \mathrm{z}^{+}}\right)=v \frac{\partial^{2} \mathrm{v}^{+}}{\partial \mathrm{z}^{+2}}-\frac{\sigma \mu_{\mathrm{e}}^{2} \mathrm{H}_{0}^{2} \sin ^{2} \alpha}{\rho} \frac{\left(m \mathrm{u}^{+} \sin \alpha+\mathrm{v}^{+}\right)}{\left(1+\mathrm{m}^{2} \sin ^{2} \alpha\right)}- \\
v^{+} \frac{v}{K^{+}} \ldots \ldots . .
\end{gathered}
$$

\subsection{Energy Equation}

$$
\frac{\partial T^{+}}{\partial t^{+}}-w_{0}^{+} \frac{\partial T^{+}}{\partial z^{+}}=\frac{k}{\rho C_{p}} \frac{\partial^{2} T^{+}}{\partial z^{+2}}-\frac{1}{\rho C_{p}} \frac{\partial q_{r}^{+}}{\partial z^{+}}
$$

Where $u^{+}, v^{+}, v, \rho, \sigma, \mathrm{m}=$ $\omega_{e} \tau_{e}, \omega_{e}, \tau_{e}, g, \beta_{T}, T^{+}, T_{\alpha}^{+}, k, C_{p} \mu_{e}, e, \eta_{e}, p_{e}$ are fluid velocity in $\mathrm{x}^{+}$-direction, fluid velocity in $\mathrm{y}^{+}$-direction, kinematic coefficient of viscosity, fluid density, electrical conductivity, Hall current parameter, cyclotron frequency, electron collision time, acceleration due to gravity, volumetric coefficient of thermal expansion, fluid temperature, temperature of the ambient fluid, Thermal conductivity of the fluid, specific heat at constant pressure, the magnetic permeability, the electric charge, the number density of electron, the electron pressure respectively

Equations (2), (3), and (4) are subject to the following initial and boundary conditions.

$$
\begin{gathered}
t^{+} \leq 0: u^{+}=0, \mathrm{v}^{+}=0, T^{+}=T_{\infty}^{+} \forall z^{+} \\
t^{+}>0: u^{+}=U_{0} t^{+2}, \mathrm{v}^{+}=0, T_{\infty}^{+}+\left(T_{w}^{+}+T_{\infty}^{+}\right) A t^{+}, \text {at } \\
z^{+}=0 \\
t^{+}>0: u^{+} \rightarrow 0 \mathrm{v}^{+} \rightarrow 0: T^{+} \rightarrow T_{\infty}^{+} \text {as } z^{+} \rightarrow \infty
\end{gathered}
$$

We now use Rosseland approximation which leads to the value of radiative heat flux as $q_{r}^{+}$

$$
q_{r}^{+}=-\frac{4 \sigma^{+}}{3 \mathrm{k}^{+}} \frac{\partial T^{+2}}{\partial z^{+}}
$$

Where $\mathrm{k}^{+}$is mean absorption coefficient and $\sigma^{+}$is StefanBoltzmann constant. It may be noted that by using Rosseland approximation we limit our analysis to optically thick fluids. Assuming small temperature differences between fluid temperature $T^{+}$and free stream temperature $T_{\alpha}^{+}$, the Eq. (4) is linearized by expanding $T^{+4}$ in Taylors series about free stream temperature $T_{\alpha}^{+}$, after neglecting second and higher order terms in $\left(T^{+}-T_{\propto}^{+}\right)$it takes the for

$$
T^{+4} \cong 4 T_{\propto}^{+3} T^{+}-3 T_{\propto}^{+4}
$$

By using equations (6) and (7), equation (4) reduces to

$$
\frac{\partial T^{+}}{\partial t^{+}}-w_{0}^{+} \frac{\partial T^{+}}{\partial z^{+}}=\frac{k}{\rho C_{p}} \frac{\partial^{2} T^{+}}{\partial z^{+2}}+\frac{16 \sigma^{+} T_{\propto}^{+3}}{\rho C_{p} 3 \mathrm{k}^{+}} \frac{\partial^{2} T^{+}}{\partial z^{+2}} \ldots .
$$

We introduce the following usual similarities

$$
\begin{gathered}
u=u^{+} /\left(\mathrm{v}^{2} U_{0}\right)^{1 / 5} \mathrm{v}=\mathrm{v}^{+} /\left(\mathrm{v}^{2} U_{0}\right)^{1 / 5}, w_{0}=w_{0}^{+} /\left(\mathrm{v}^{2} U_{0}\right)^{1 / 5}, K \\
=K^{+}\left(\frac{U_{0}^{2}}{\mathrm{v}^{6}}\right)^{1 / 5} z=z^{+}\left(U_{0} / \mathrm{v}^{3}\right)^{1 / 5}, \\
t=t^{+}\left(U_{0}^{2} / \mathrm{v}\right)^{1 / 5} P_{r}=\frac{v \rho \mathrm{C}_{\mathrm{p}}}{k}, G r=\frac{\beta_{T} \rho\left(T_{w}^{+}-T_{\infty}^{+}\right)}{\left(v U_{0}^{3}\right)^{1 / 5}}, \theta=\frac{T^{+}-T_{\alpha}^{+}}{T_{w}^{+}-T_{\alpha}^{+\prime}} \\
\mathrm{M}=\frac{\sigma}{\rho(} \frac{\mu_{e}^{2} H_{0}^{2} \psi^{2}}{\left.m_{*}^{2}+1\right)}\left(v^{2} \mathrm{U}_{0}\right)^{1 / 5}, F=\frac{4 T_{\alpha}^{+3}}{\mathrm{kk}^{+}}
\end{gathered}
$$

where Gr, M, Pr, R, and $K$ are thermal Grashof number, the magnetic parameter, Prandtl number, radiation parameter and permeability of porous medium, respectively.

Equations (2), (3), and (8) reduce to the following dimensionless form:

$$
\begin{gathered}
\left(\frac{\partial u}{\partial t}-w_{0} \frac{\partial u}{\partial z}\right)=\frac{\partial^{2} u}{\partial z^{2}}+\operatorname{Gr} \theta \cos \alpha-\left(\frac{M^{2} \sin ^{2} \alpha}{1+m^{2} \sin ^{2} \alpha}+\frac{1}{K}\right) u+ \\
\frac{m M^{2}}{1+m^{2} \sin ^{2} \alpha} v \\
\left(\frac{\partial \mathrm{v}}{\partial t}-w_{0} \frac{\partial \mathrm{v}}{\partial z}\right)=\frac{\partial^{2} \mathrm{v}}{\partial z^{2}}-\left(\frac{M^{2} \sin ^{2} \alpha}{1+m^{2} \sin ^{2} \alpha}+\frac{1}{K}\right) v-\frac{m M^{2}}{1+m^{2} \sin ^{2} \alpha} u \\
\left(\frac{\partial \theta}{\partial t}-\mathrm{w}_{0} \frac{\partial \theta}{\partial z}\right)=\frac{1}{P_{r}}\left(1+\frac{4 R}{3}\right) \frac{\partial^{2} \theta}{\partial z^{2}}
\end{gathered}
$$

Also the boundary conditions become

$$
\left.\begin{array}{c}
t \leq 0: u=0 \mathrm{v}=0 \theta=0, \forall z \\
t>0: u=t^{2} \mathrm{v}=0 \theta=t, \text { at } z=0 \\
t>0: u \rightarrow 0 \mathrm{v} \rightarrow 0 T \rightarrow 0 \text { as } \mathrm{z} \rightarrow \infty
\end{array}\right\}
$$

\section{Numerical Procedure}

Equations governing the flow are highly non-linear. Getting an exact analytical solution to them is not possible. We generate numerical solutions of the equations by using the finite difference method of Crank-Nicolson Method. The equations are solved subject to the initial and boundary conditions. The equivalent finite difference scheme of equations (9), (10) and (11) is as follows

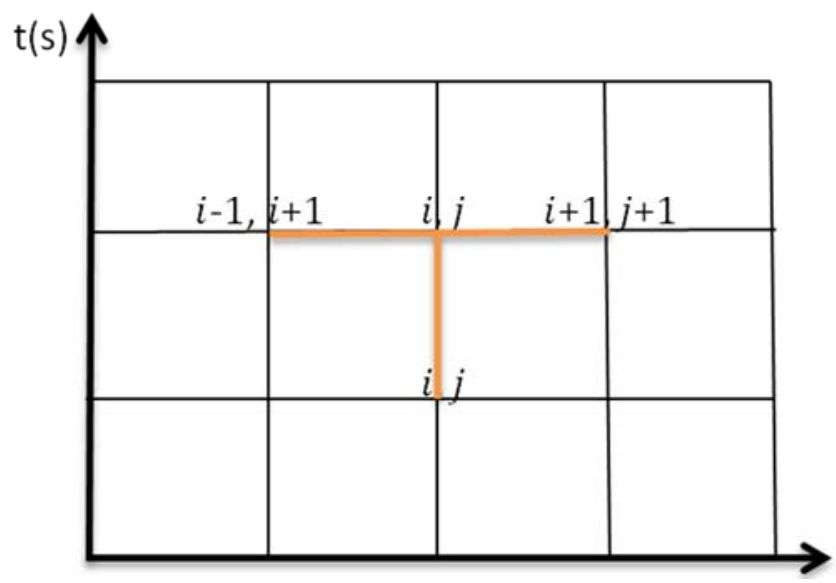

Figure 2. Explicit Finite Difference System Grid. 


$$
\begin{gathered}
\frac{u_{i}^{j+1}-u_{i}^{j}}{\Delta t}-\mathrm{w}_{0}\left(\frac{u_{i+1}^{j+1}-u_{i-1}^{j+1}+u_{i+1}^{j}-u_{i-1}^{j}}{4 \Delta \mathrm{z}}\right)=\left(\frac{u_{i+1}^{j+1}-2 u_{i}^{j+1}+u_{i-1}^{j+1}+u_{i+1}^{j}-2 u_{i}^{j}+u_{i-1}^{j}}{2(\Delta \mathrm{z})^{2}}\right)+\operatorname{Gr} \cos \alpha \theta_{i}^{j}-\left(\frac{M^{2} \sin ^{2} \alpha}{1+m^{2} \sin ^{2} \alpha}+\frac{1}{K}\right)\left(\frac{u_{i}^{j+1}+u_{i}^{j}}{2}\right)+\frac{\mathrm{mM}^{2}}{1+m^{2} \sin ^{2} \alpha} v_{i}^{j} \\
\frac{v_{i}^{j+1}-v_{i}^{j}}{\Delta t}-\mathrm{w}_{0}\left(\frac{v_{i+1}^{j+1}-v_{i-1}^{j+1}+v_{i+1}^{j}-v_{i-1}^{j}}{4 \Delta \mathrm{z}}\right)=\left(\frac{v_{i+1}^{j+1}-2 v_{i}^{j+1}+v_{i-1}^{j+1}+v_{i+1}^{j}-2 v_{i}^{j}+v_{i-1}^{j}}{2(\Delta \mathrm{z})^{2}}\right)-\left(\frac{M^{2} \sin ^{2} \alpha}{1+m^{2} \sin ^{2} \alpha}+\frac{1}{K}\right)\left(\frac{v_{i}^{j+1}+v_{i}^{j}}{2}\right)-\frac{\mathrm{mM}^{2}}{1+m^{2} \sin ^{2} \alpha} u_{i}^{j} \cdots \\
\frac{\theta_{i}^{j+1}-\theta_{i}^{j}}{\Delta t}-\mathrm{w}_{0}\left(\frac{\theta_{i+1}^{j+1}-\theta_{i-1}^{j+1}+\theta_{i+1}^{j}-\theta_{i-1}^{j}}{4 \Delta \mathrm{z}}\right)=\frac{1}{P r}\left(1+\frac{4 R}{3}\right)\left(\frac{\theta_{i+1}^{j+1}-2 \theta_{i}^{j+1}+\theta_{i-1}^{j+1}+\theta_{i+1}^{j}-2 \theta_{i}^{j}+\theta_{i-1}^{j}}{2(\Delta \mathrm{z})^{2}}\right) \cdots
\end{gathered}
$$

Multiplying through by $4 \Delta t(\Delta \mathrm{z})^{2}$ we get from equation (13)

$$
\begin{array}{r}
4(\Delta \mathrm{z})^{2}\left(u_{i}^{j+1}-u_{i}^{j}\right)-\mathrm{w}_{0} \Delta t \Delta \mathrm{z}\left(u_{i+1}^{j+1}-u_{i-1}^{j+1}+u_{i+1}^{j}-u_{i-1}^{j}\right)=2 \Delta t\left(u_{i+1}^{j+1}-2 u_{i}^{j+1}+u_{i-1}^{j+1}+u_{i+1}^{j}-2 u_{i}^{j}+u_{i-1}^{j}\right)+ \\
4 \Delta t(\Delta \mathrm{z})^{2} G r \cos \alpha \theta_{i}^{j}-2 \Delta t(\Delta \mathrm{z})^{2}\left(\frac{M^{2} \sin ^{2} \alpha}{1+m^{2} \sin ^{2} \alpha}+\frac{1}{K}\right)\left(u_{i}^{j+1}+u_{i}^{j}\right)+4 \Delta t(\Delta \mathrm{z})^{2} \frac{\mathrm{mM}^{2}}{1+m^{2} \sin ^{2} \alpha} v_{i}^{j}
\end{array}
$$

If we let the coefficients of interior nodes to be:

$$
\begin{gathered}
a_{i}=\left(\mathrm{w}_{0} \Delta t \Delta \mathrm{z}-2 \Delta t\right) \\
b_{i}=\left(4(\Delta z)^{2}+4 \Delta t+2 \Delta t(\Delta z)^{2}\left(\frac{M^{2} \sin ^{2} \alpha}{1+m^{2} \sin ^{2} \alpha}+\frac{1}{K}\right)\right) \\
c_{i}=\left(-\mathrm{w}_{0} \Delta t \Delta \mathrm{z}-2 \Delta t\right) \\
d_{i}=u_{i-1}^{j}\left(-\mathrm{w}_{0} \Delta t \Delta \mathrm{z}+2 \Delta t\right) \text { For } i=2,3, \ldots, N-1 \\
e_{i}=u_{i}^{j}\left(4(\Delta z)^{2}-4 \Delta t-2 \Delta t(\Delta z)^{2}\left(\frac{M^{2} \sin ^{2} \alpha}{1+m^{2} \sin ^{2} \alpha}+\frac{1}{K}\right)\right) \\
g=\left[4 \Delta t(\Delta \mathrm{z})^{2} \operatorname{Gr} \cos \alpha \theta_{i}^{j}+4 \Delta t(\Delta \mathrm{z})^{2} \frac{\mathrm{mM}^{2}}{1+m^{2} \sin ^{2} \alpha} v_{i}^{j}\right]
\end{gathered}
$$

Therefore equation (16) becomes:

$$
a_{i} u_{i-1}^{j+1}+b_{i} u_{i}^{j+1}+c_{i} u_{i+1}^{j+1}=d_{i}+e_{i}+f_{i}+g
$$

If we let $n=i+1$

For $i=2$ equation (18) becomes

$$
a_{2} u_{1}^{n}+b_{2} u_{2}^{n}+c_{2} u_{3}^{n}=d_{2}+e_{2}+f_{2}+g
$$

For $i=3$ equation (18) becomes

$$
a_{3} u_{2}^{n}+b_{3} u_{3}^{n}+c_{3} u_{4}^{n}=d_{3}+e_{3}+f_{3}+g
$$

For $i=4$ equation (18) becomes

$$
a_{4} u_{3}^{n}+b_{4} u_{4}^{n}+c_{4} u_{5}^{n}=d_{4}+e_{4}+f_{4}+g
$$

If we proceed up to $i=N-1$, the system of equations can be represented in matrix form as:

$$
\left.\left[\begin{array}{cccccc}
a_{2} & b_{2} & c_{2} & 0 & \cdots & 0 \\
0 & a_{3} & b_{3} & c_{3} & 0 & \vdots \\
\vdots & 0 & \ddots & \ddots & \ddots & 0 \\
0 & 0 & 0 & \ddots a_{N-1} & \cdot b_{N-1} & \cdot c_{N-1}
\end{array}\right] \begin{array}{c}
u_{1}^{n} \\
u_{2}^{n} \\
\vdots \\
u_{N-1}^{n}
\end{array}\right]=\left[\begin{array}{c}
d_{2} \\
d_{3} \\
\vdots \\
d_{N-1}
\end{array}\right]+\left[\begin{array}{c}
e_{2} \\
e_{3} \\
\vdots \\
e_{N-1}
\end{array}\right]+\left[\begin{array}{c}
f_{2} \\
f_{3} \\
\vdots \\
f_{N-1}
\end{array}\right]+\left[\begin{array}{c}
g \\
g \\
\vdots \\
g
\end{array}\right]
$$

Also for equation (14) the interior notes are; 


$$
\begin{gathered}
a_{i}=\left(\mathrm{w}_{0} \Delta t \Delta \mathrm{z}-2 \Delta t\right) \\
b_{i}=\left(4(\Delta z)^{2}+4 \Delta t+2 \Delta t(\Delta z)^{2}\left(\frac{M^{2} \sin ^{2} \alpha}{1+m^{2} \sin ^{2} \alpha}+\frac{1}{K}\right)\right) \\
c_{i}=\left(-\mathrm{w}_{0} \Delta t \Delta \mathrm{z}-2 \Delta t\right) \\
d_{i}=\mathrm{v}_{i-1}^{j}\left(-\mathrm{w}_{0} \Delta t \Delta \mathrm{z}+2 \Delta t\right) \text { For } i=2,3, \ldots, N-1 \\
e_{i}=\mathrm{v}_{i}^{j}\left(4(\Delta z)^{2}-4 \Delta t-2 \Delta t(\Delta z)^{2}\left(\frac{M^{2} \sin ^{2} \alpha}{1+m^{2} \sin ^{2} \alpha}+\frac{1}{K}\right)\right) \\
f_{i}=\mathrm{v}_{i+1}^{j}\left(\mathrm{w}_{0} \Delta t \Delta \mathrm{z}+2 \Delta t\right) \\
g=\left[-4 \Delta t(\Delta \mathrm{z})^{2} \frac{\mathrm{mM}^{2}}{1+m^{2} \sin ^{2} \alpha} u_{i}^{j}\right]
\end{gathered}
$$

Also for equation (15) the interior notes are;

$$
\begin{gathered}
a_{i}=\left(\mathrm{w}_{0} \Delta t \Delta \mathrm{z}-2 \Delta t\right) \\
b_{i}=\left(4(\Delta z)^{2}+\frac{4 \Delta t}{P r}\left(1+\frac{4 R}{3}\right)\right) \\
c_{i}=\left(-\mathrm{w}_{0} \Delta t \Delta \mathrm{z}-2 \Delta t\right) \\
d_{i}=\theta_{i-1}^{j}\left(-\mathrm{w}_{0} \Delta t \Delta \mathrm{z}+2 \Delta t\right) \text { For } i=2,3, \ldots, N-1 \\
e_{i}=\theta_{i}^{j}\left(\left(4(\Delta z)^{2}-\frac{4 \Delta t}{P r}\left(1+\frac{4 R}{3}\right)\right)\right) \\
f_{i}=\theta_{i+1}^{j}\left(\mathrm{w}_{0} \Delta t \Delta \mathrm{z}+2 \Delta t\right)
\end{gathered}
$$

With matrix representation as

$$
\left[\begin{array}{cccccc}
a_{2} & b_{2} & c_{2} & 0 & \cdots & 0 \\
0 & a_{3} & b_{3} & c_{3} & 0 & \vdots \\
\vdots & 0 & \ddots & \ddots & \ddots & 0 \\
0 & 0 & 0 & \ddots a_{N-1} & \ddots b_{N-1} & \ddots \\
c_{N-1}
\end{array}\right]\left[\begin{array}{c}
u_{1}^{n} \\
u_{2}^{n} \\
\vdots \\
u_{N-1}^{n}
\end{array}\right]=\left[\begin{array}{c}
d_{2} \\
d_{3} \\
\vdots \\
d_{N-1}
\end{array}\right]+\left[\begin{array}{c}
e_{2} \\
e_{3} \\
\vdots \\
e_{N-1}
\end{array}\right]+\left[\begin{array}{c}
f_{2} \\
f_{3} \\
\vdots \\
f_{N-1}
\end{array}\right]
$$

Initial and boundary conditions takes the following form

$$
\begin{gathered}
u_{(i)}^{0}=0, v_{(i)}^{0}=0, \theta_{(i)}^{0}=0 \text { for all } i \\
u_{(0, i)}^{j}=(j \Delta t)^{2}, v_{(0, i)}^{j}=0, \theta_{(0, i)}^{j}=j \Delta t \\
u_{(N)}^{j}=0, v_{(N)}^{j}=0, \theta_{(N)}^{j}=0
\end{gathered}
$$

Where $\mathrm{N}$ corresponds to $\infty$. The suffix $i$ and $j$ corresponds to $z$ and $t$ respectively. Also $\Delta t=t_{j+1}-t_{j}$ and $\Delta z=z_{i+1}-$ $z_{i}$

\section{Result and Discussion}

The velocity and Temperature profiles have been computed by using Crank- Nicolson finite difference method. We have presented the non-dimensional velocity components and temperature distribution for several values of magnetic parameter $M^{2}$, radiation parameter $R$, Hall parameter $m$, Prandtl number $\mathrm{Pr}$, Grashof number $\mathrm{Gr}$ and time t in Figures. 3 to 12 Figures. 3(a) and (b) to 9(a) and (b) represent the primary velocity $u$ and secondary velocity $v$ against $\mathrm{z}$ for several values of $M^{2}, R, m, P r, G r$ and t. Figures 10 to 12 show the temperature profiles. 11 show the temperature profiles. Computation are performed with small values of $\Delta t=0.01$. We set $z_{0}=$ $0, Z_{N}=1$ where $\mathrm{N}$ correspond to $\infty, n z=100$ corresponds to $\mathrm{z}=\infty, t_{0}=0$, and $\Delta x=\Delta z=0.01$. We hence fix $u_{(100, i)}^{n}=v_{(100, i)}^{n}=\theta_{(100, i)}^{n}=0$. As $u, \mathrm{v}$ and $\theta$ tends to zero when $z=100$. the following parameters are fixed throughout the calculation except where otherwise stated, $M^{2}=1, R=5, m=0.5, P r=0.71, G r=5 \alpha=\pi / 4, \mathrm{~K}=0.1$, and $\mathrm{t}=0.5$. 


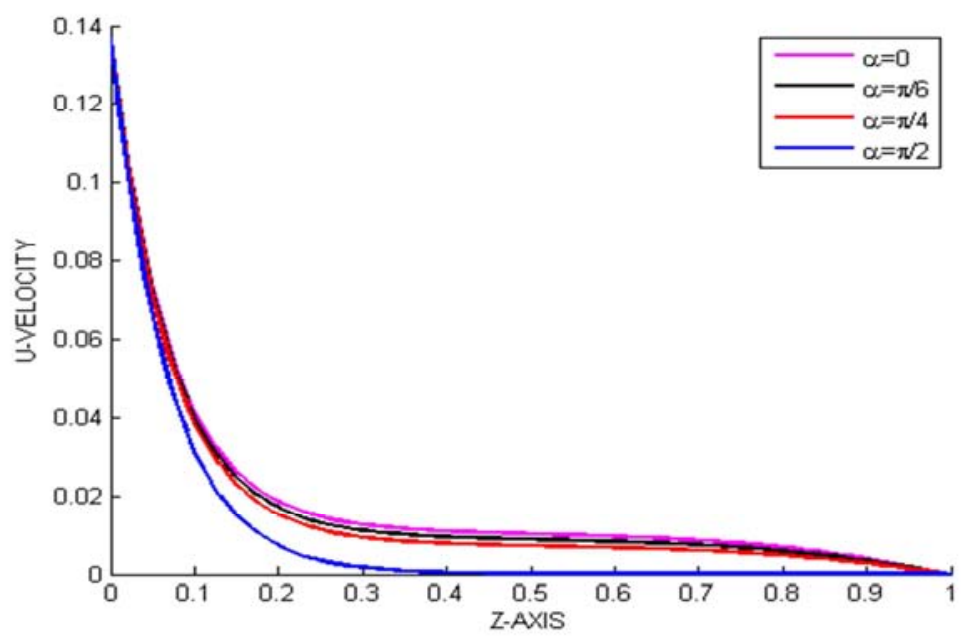

3(a)

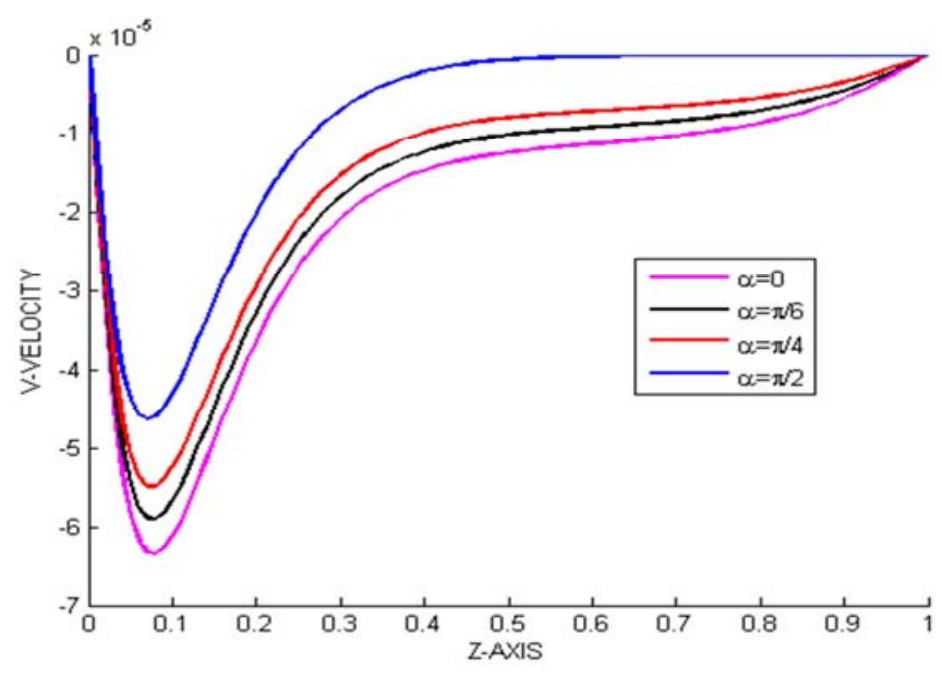

3(b)

Figure 3. (a) and (b) Effect of angle of inclination ( $\alpha$ ): The velocity profile for different values of angle inclination $(\alpha=0, \pi / 6, \pi / 4, \pi / 2)$ when $M^{2}=1.0, R=5$, $m=0.5, \operatorname{Pr}=0.71, G r=5, K=1.0$ and $t=0.5$.

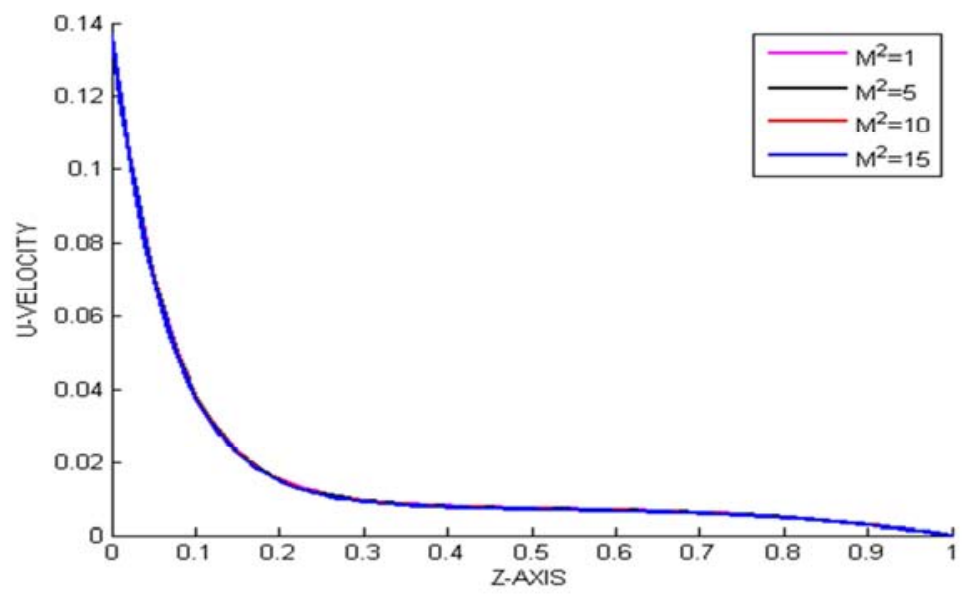

4(a) 


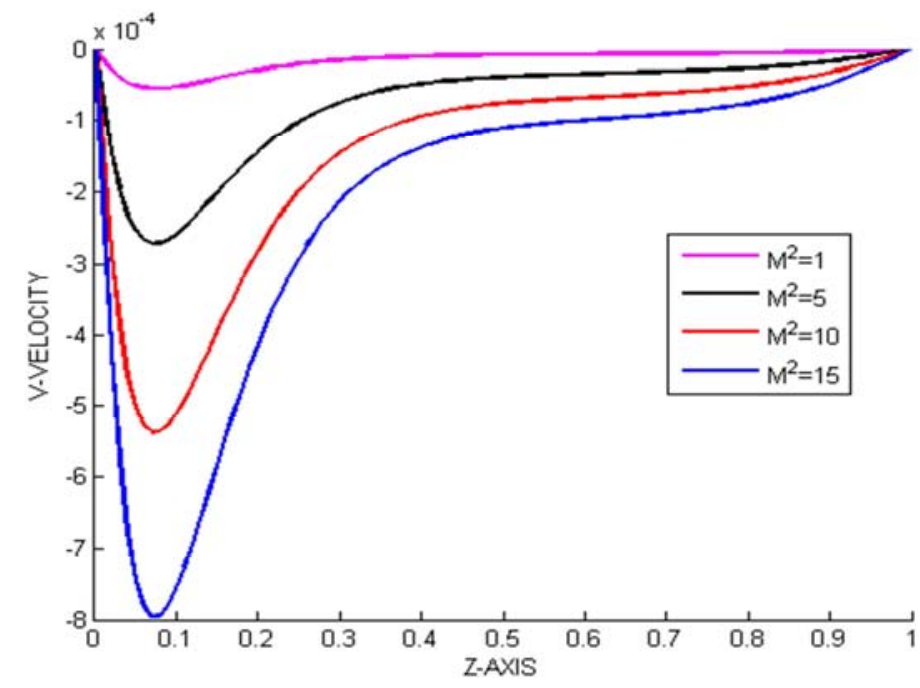

4(b)

Figure 4. (a) and (b) Effect of magnetic field $M^{2}$ : The velocity profile for different values of magnetic field $\left(M^{2}=1,5,10,15\right)$ when, $R=4, m=0.5, P r=0.71$, $G r=5, t=0.5, K=1.0$ and $\alpha=\pi / 4$.

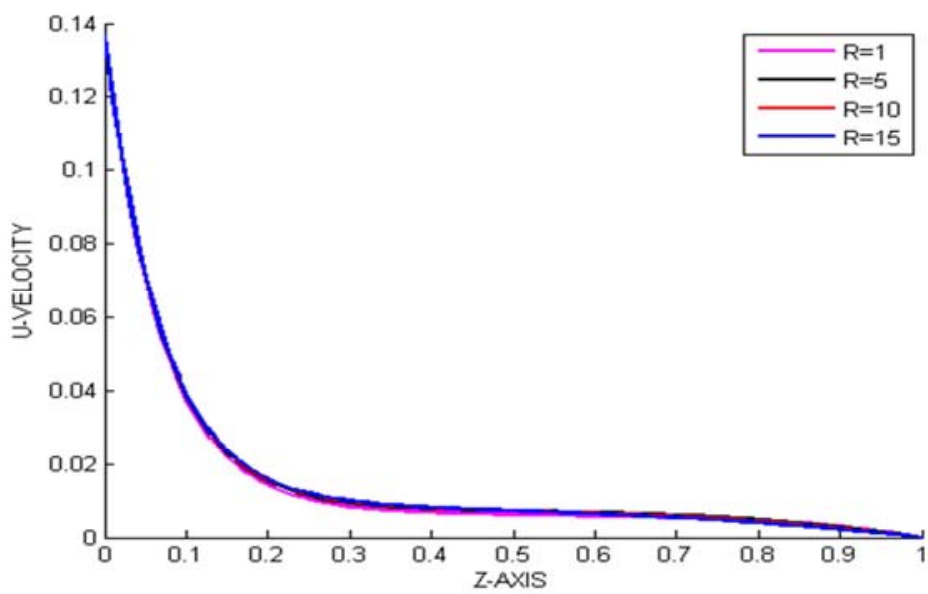

(5a)

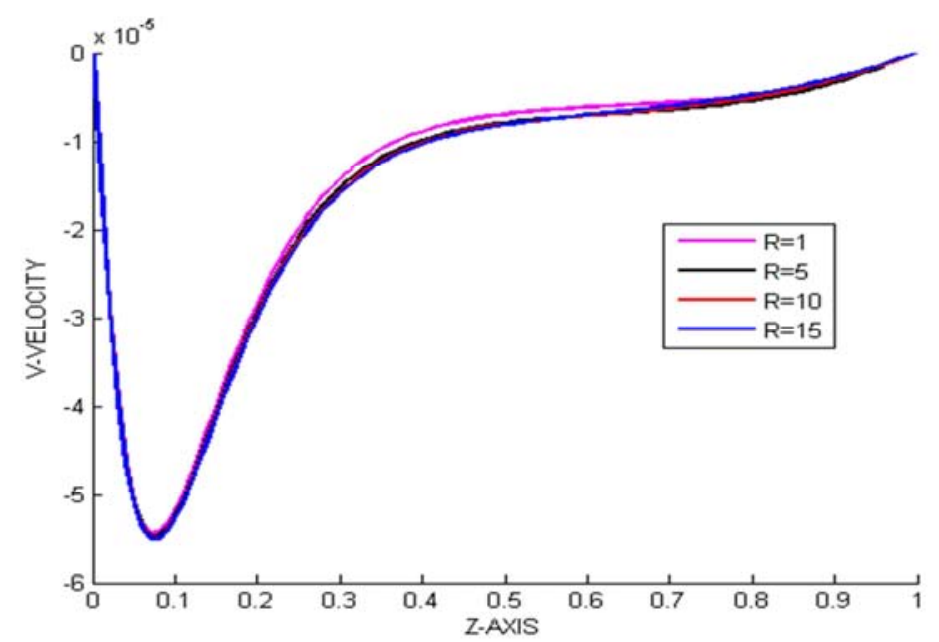

(5b)

Figure 5 (a) and (b) Effect of radiation parameter. $R$ : The velocity profile for different values of radiation parameter $(R=1,5,10,15)$ when, $M^{2}=1, m=0.5$, $P r=0.71, G r=5, t=0.5, K=1.0$ and $\alpha=\pi / 4$. 


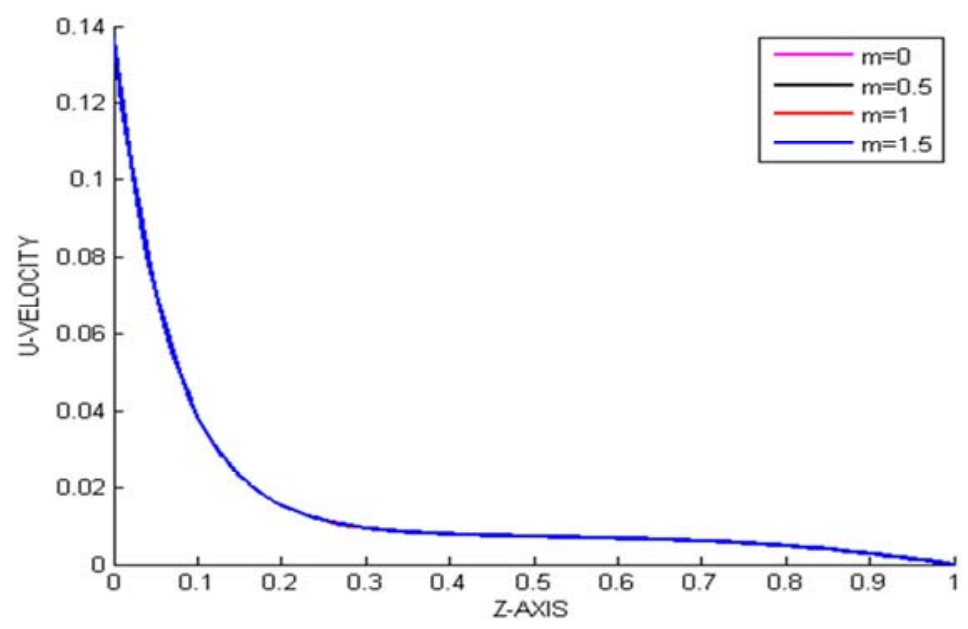

(6a)

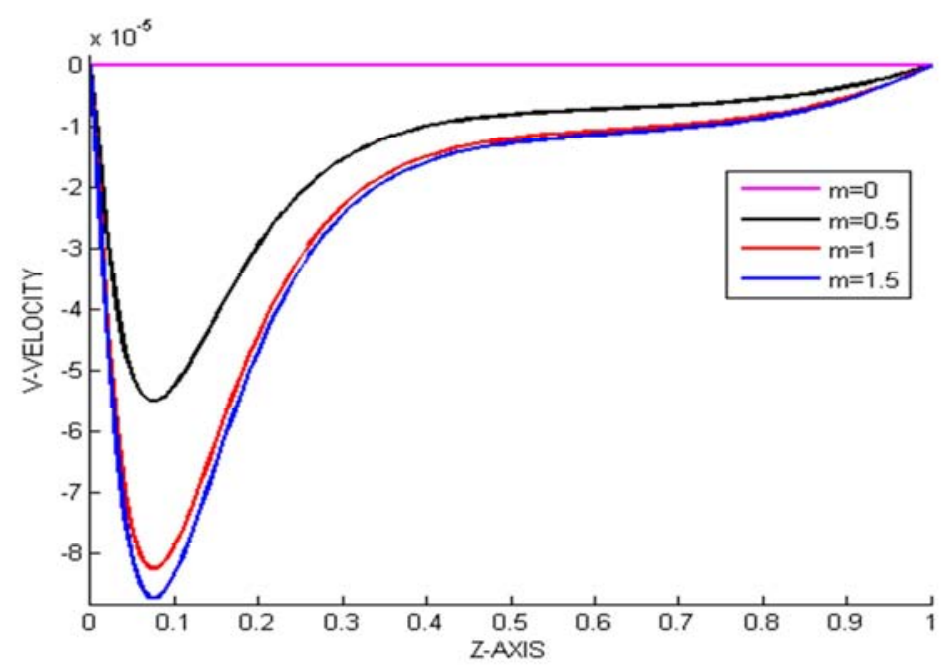

(6b)

Figure 6. (a) and (b) Effect of hall current. $m$ : The velocity profile for different values hall current parameter. $(m=0, \pi / 6, \pi / 4, \pi / 2)$ when, $M^{2}=1, R=5$, $\operatorname{Pr}=0.71, G r=5, t=0.5, K=1.0$ and $\alpha=\pi / 4$.

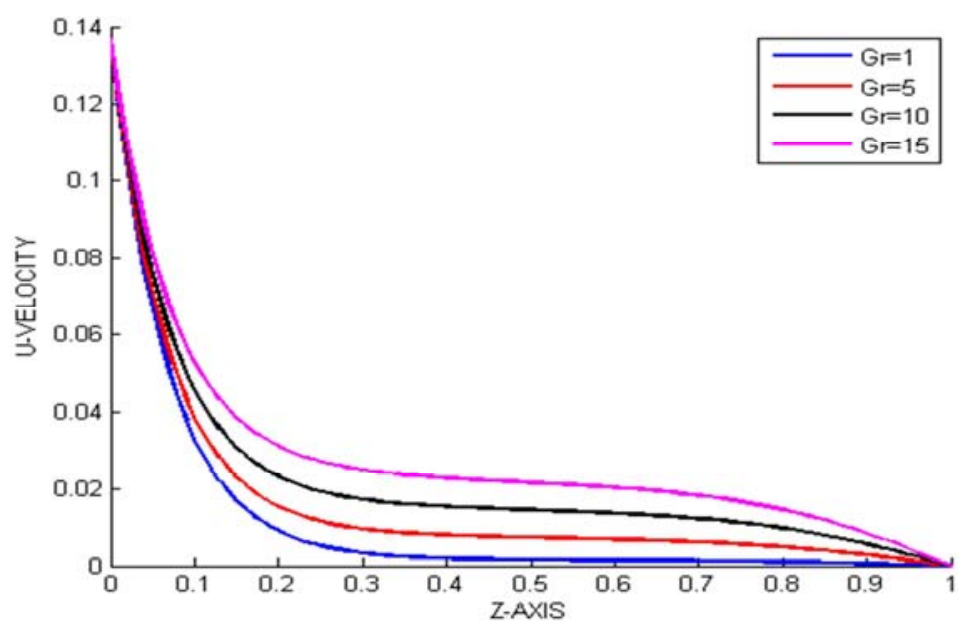




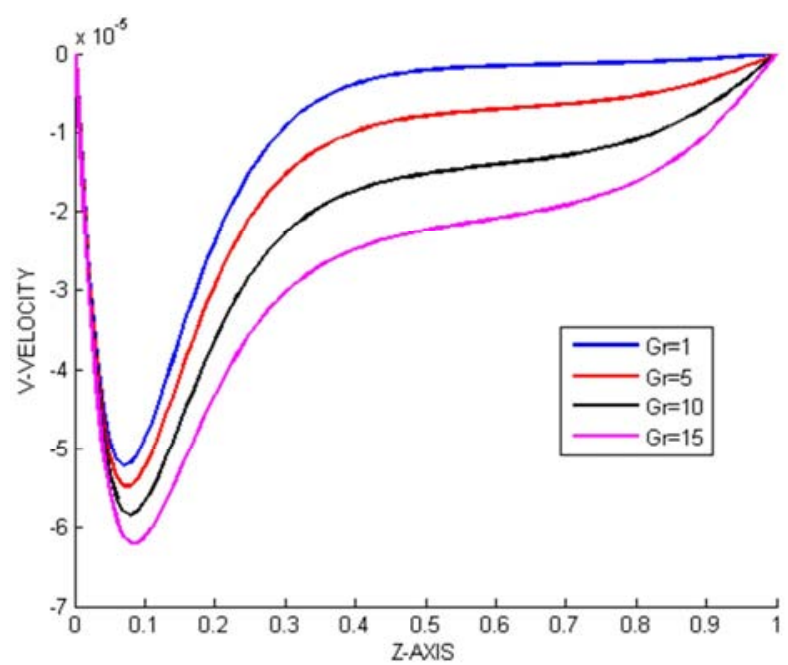

(7b)

Figure 7. (a) and (b) Effect of Grashof number Gr: The velocity profile for different $G r$. (Gr=1,5,10,15, ) when, $M^{2}=5, m=0.5, R=5, P r=0.71, t=0.5, K=1.0$ and $\alpha=\pi / 4$.

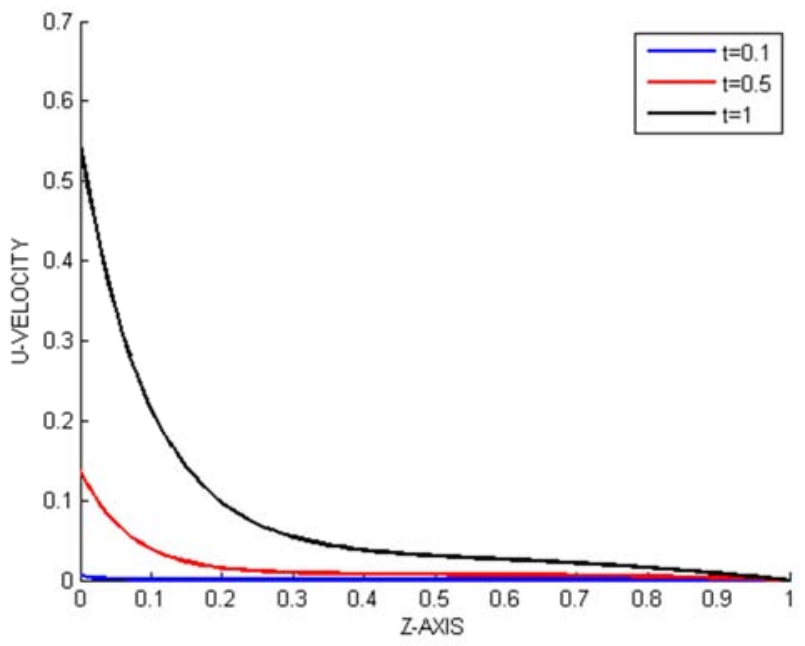

8(a)

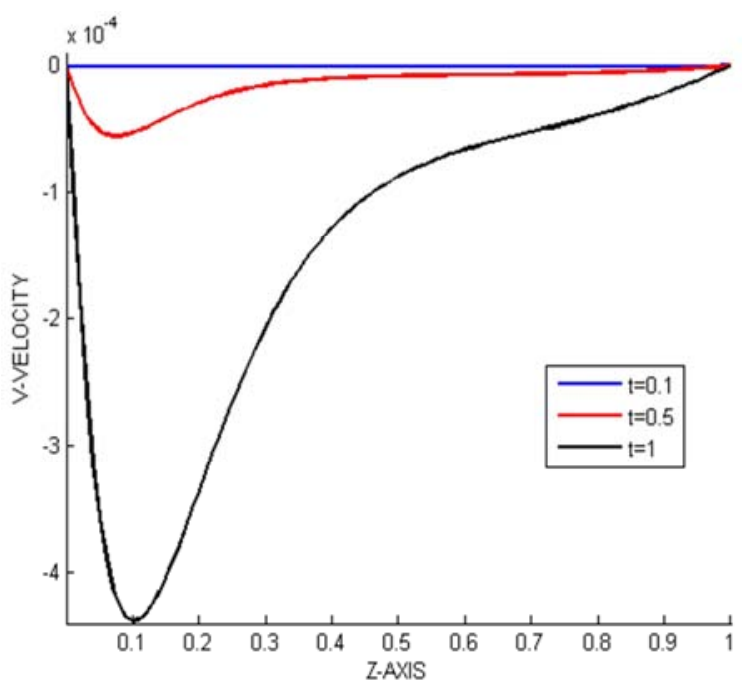

(8b)

Figure 8. (a) and (b) Effect of time $t$ : The velocity profile for different time $t$. $(t=0.1,0.5,1.0,1.5)$ when, $M^{2}=5, m=0.5, R=4, G r=5, P r=0.71, t=0.5, K=1.0$ and $\alpha=\pi / 4$. 


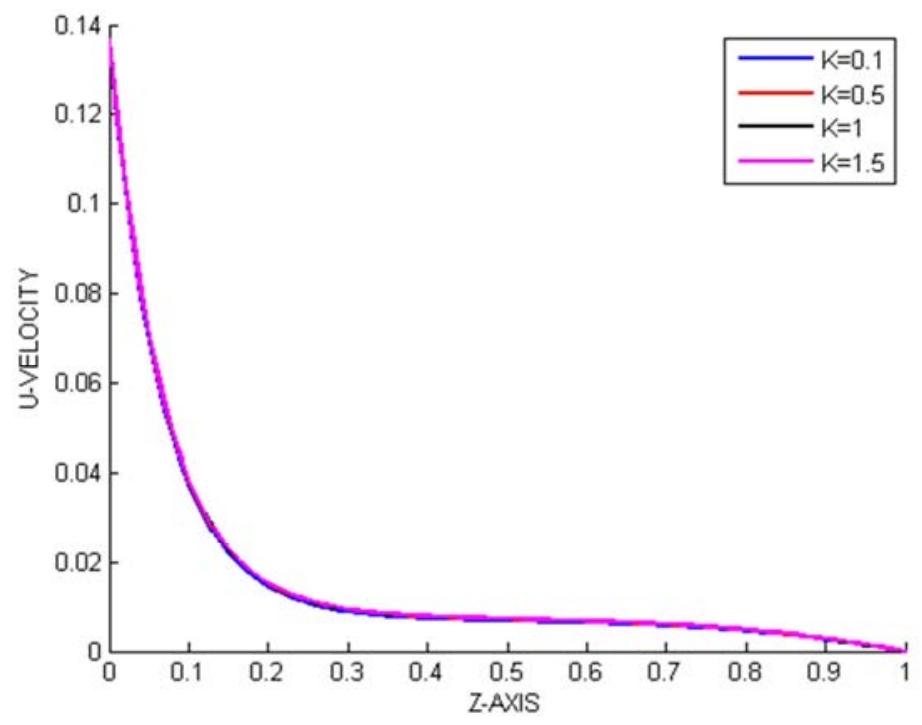

(9a)

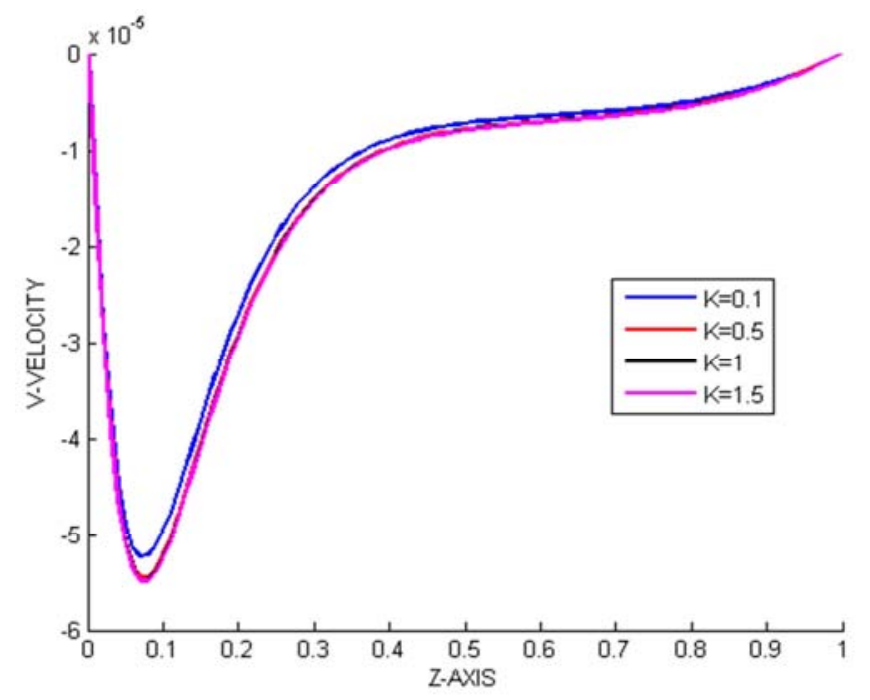

(9b)

Figure 9. (a) and (b) Effect of permeability parameter $K$,: The permeability parameter $K$. $(K=0.1,0.5,1.0,1.5)$ when, $M^{2}=5, m=0.5, R=5, G r=5, P r=0.71$, $t=0.5$, and $\alpha=\pi / 4$.

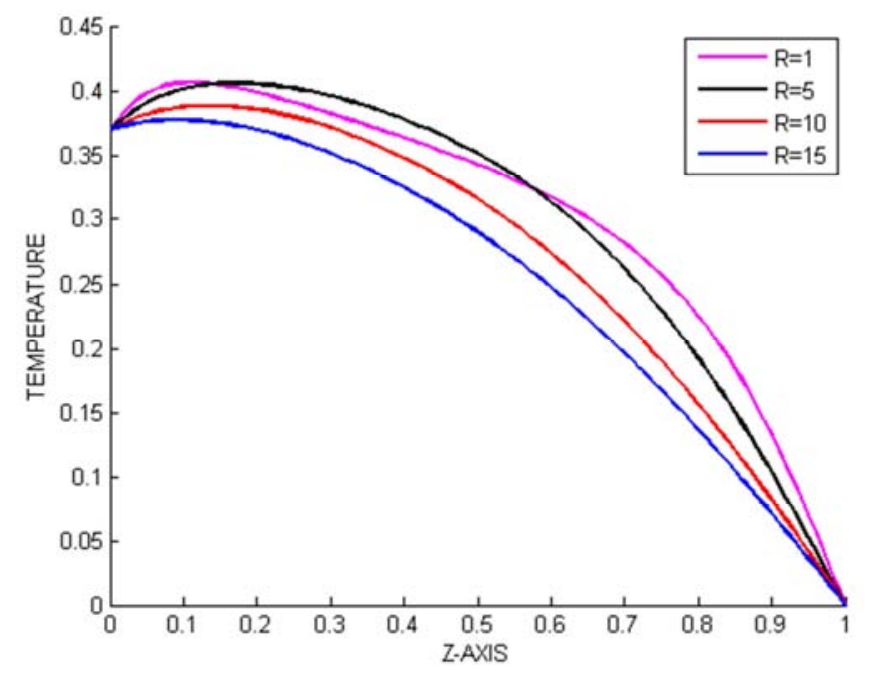

Figure 10. Temperature profile for $R .(R=1,5,10,15)$ when, $\operatorname{Pr}=0.71$, and $t=0.5$. 


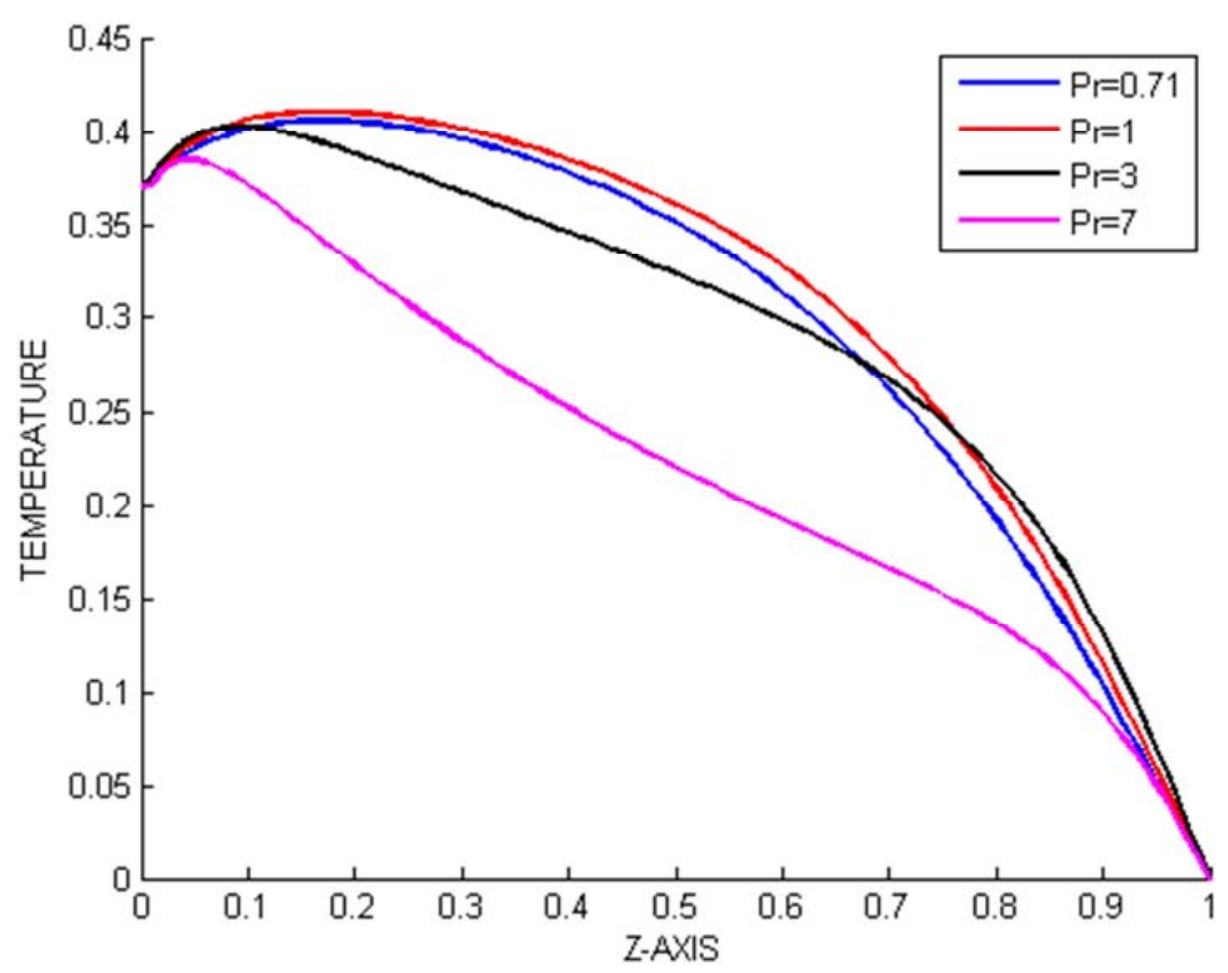

Figure 11. Temperature profile for $\operatorname{Pr}$. $(\operatorname{Pr}=0.71,1.0,3.0,7.0)$ when, $R=4$, and $t=0.5$.

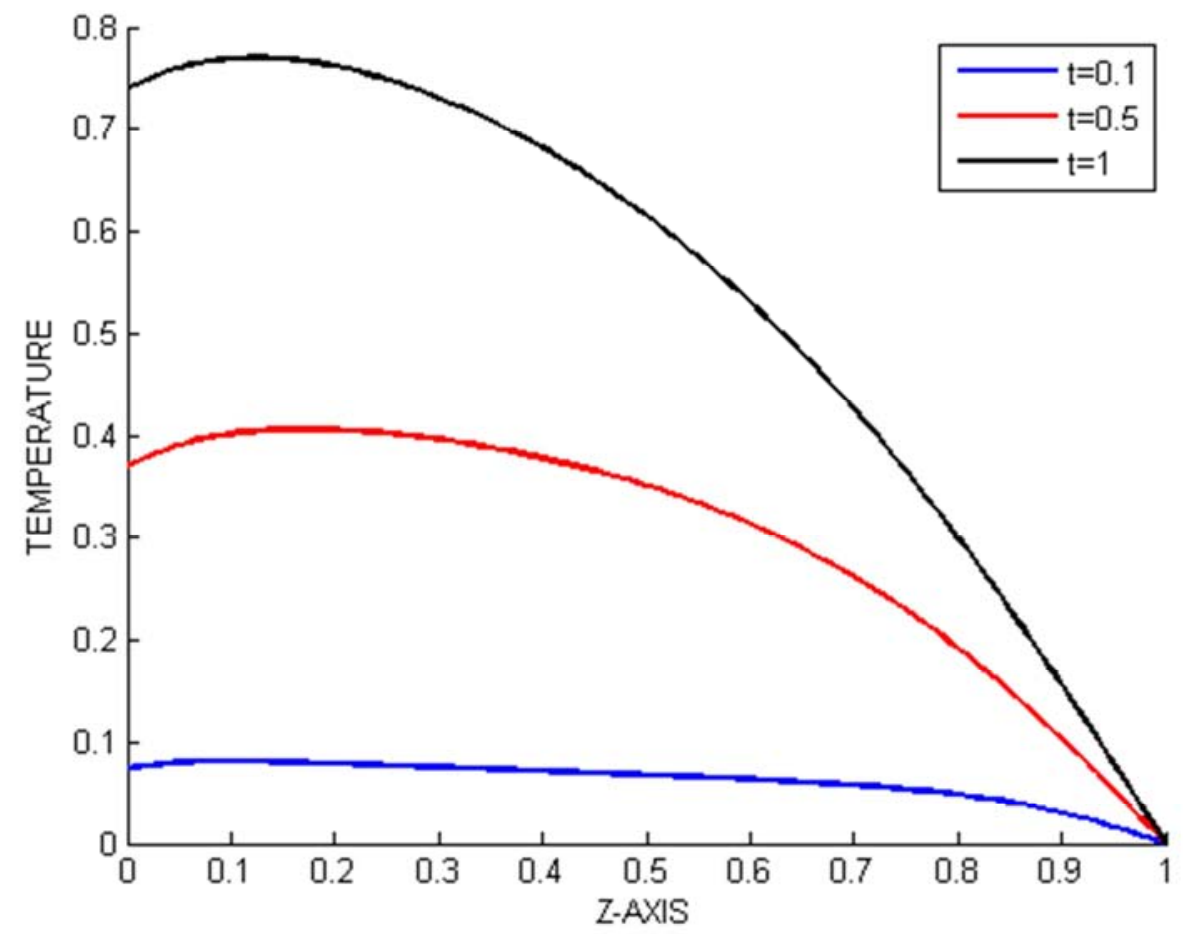

Figure 12. Temperature profile for $t$. $(t=0.1,0.5,1.0,1.5)$ when, $r=0.71$, and $t=0.5$.

In order to get physical insight into the problem under study, the velocity field and temperature field are discussed by assigning numerical values to the parameters i.e. the angle of inclination and non - dimensional numbers (Hall parameter, Radiation, and heat parameter) encountered into the corresponding Equations. The velocities are classified as primary $(\mathrm{u})$ and secondary $(\mathrm{v})$ along the $\mathrm{x}$ and $\mathrm{z}$ axes respectively.

a) Velocity

i) It is observed that when the plate is inclined at angle $\alpha$ it affects the applied magnetic field; hence the angle of inclination of the plate is equal to the angle made by the transverse magnetic field and the inclined plate. From figure (3a) it is observed 
that increase in angle of inclination leads to a decrease in the primary velocity while in figure (3b) shows that an increase in the angle of inclination leads to an increase in the secondary velocity.

ii) Figure (4a) it observed that the primary velocity remains constant as the Hartmann number increases while figure (4b) illustrates that increase in Hartmann number decreases the secondary velocity. Hartmann number is a ratio of magnetic force to viscous force.

iii) Figure (5a) and (b) shows that the radiation parameter does not affect the flow of fluid i.e as the radiation parameter increases the primary and secondary velocity remains constant.

iv) Figure (6a) it observed that as the primary velocity remains constant as the Hall current parameter increases while figure (6b) as the Hall current increases the secondary velocity decreases.

v) From figure (7a) it is observed that increase in the Grashof number leads to a decrease in the primary velocity while in figure (7b) shows that an the increase in the Grashof number leads to an increase in the secondary velocity.

vi) From figure ( $8 \mathrm{a})$ shows that at time $\mathrm{t}=0$ velocity is zero and as time increases the primary velocity increases while figure (8b) show that at $\mathrm{t}=0$ velocity is zero and time increases the primary velocity decreases.

vii) Figure (9a) shows that primary velocity is not affected with increasing permeability parameter while (9b) show that as permeability increases there is a slight increase in the secondary velocity.

b) Temperature profiles

viii) From figure $10 \mathrm{It}$ is observed that increase in Radiation parameter leads to a decrease in the temperature distribution in the thermal boundary layer.

ix) Figure 11. It is observed that increase value of Prandtl number implies decrease in the temperature distribution profile. This leads to thermal boundary layer thickness.

$\mathrm{x}$ ) From figure 12 It is observed that increase in time leads to the increase in the temperature profile. With time as the flow gets to the free stream the velocity is increased hence there is increased rate of energy transfer and therefore the temperature will increase.

\section{Conclusion}

The problem of two-dimensional fluid flow in the presence of hall current and radiation effects under the influence of uniform magnetic field applied normal to the flow is formulated and solved numerically. A Crank-Nicolson method with the help of MATLAB program is adopted to solve the equations governing the flow. It is found that increasing the Prandtl number results in a decrease in the temperature profile. An increase in Radiation parameter leads to a decrease in the temperature distribution in the thermal boundary layer.

From this results, it's clear that the parameters in the governing equations affect the primary, secondary and temperature profile. It's recommended that this work be extended by considering; variable transverse magnetic field, non Newtonian fluids e.g the micropolar fluids and also the effects of the parameters in the governing equations on skin friction and rate of mass transfer

\section{Acknowledgment}

Authors are grateful to Dr Mark Mwiti Kimathi for designing and coding the research work using MATLAB.

\section{References}

[1] Kirimi.J. et al (2012) studied Magnetic field and Hall current effect on MHD free convective flow past a vertical rotating plate. Asian Journal of Engineering and maths 1:6 346-354.

[2] V.Sugunamma, N.Sandeep, P.Mohan Krishna, Ramana Bahunadam (2013) Inclined magnetic field and chemical reaction effects on flow over a semi infinite vertical porous plate through porous media. Communications in Applied Sciences Vol 1 No. 1 1-24

[3] Sarkar B.C. Das.S Jana R. N.(2013) Inclined Magneticfield and Chemical Reaction Effects on Flow over a Semi Infinite Vertical Porous Plate through Porous Medium. Communications in Applied Sciences ISSN 2201-7372 Vol. 1, No.1, pp.1-24

[4] Murali Gundagani,Sivaiah Sheri, Ajit Paul and M. C.K.Reddy (2013) Unsteady Magnetohydrodynamic Free Convective Flow Past a Vertical Porous Plate. International Journal of Applied Science and Engineering Vol 3 No 11, 267-275

[5] Sigey K. Johana, Okelo A. Jeconia, Gatheri K. Francis, Ngesa O. Joel (2013) Magnetohydrodynamic (MHD) Free Convective Flow past an Infinite Vertical Porous Plate with Joule Heating. http://www.scirp.org/journal/am

[6] Rogers Omboga Amenya, Johanna Kibet Sigey, Jaconiah Abonyo Okelo, James Mariita Okwoyo (2013)MHD Free Convection Flow past a Vertical Infinite Porous Plate in the Presence of Transverse Magnetic Field with Constant Heat Flux. International Journal of science and research Vol. 2 Issue 10 pp 217-222

[7] Amos S. Idowu, Abdulwaheed Jimoh, Funmilayo H. Oyelami and Moses S. Dada (2014) Numerical solution for thermal radiation effect on inclined magnetic field of MHD free convective Heat transfer dissipative fluid flow past a moving vertical porous plate with variable suction. American Journal of Fluid Dynamics 4(3):pp 91-101

[8] A.Neel Armstrongand R. Muthucumaraswamy (2014) Mhd Flow Past A Parabolic Started Vertical Plate With Variable Temperature And Mass Diffusion Journal of Mechanical Engineering and Sciences (JMES) ISSN: 2231-8380; Vol. 7, pp.1251-1260 
[9] R. Muthucumaraswamy, and E. Geetha (2014) Effects of parabolic motion on an isothermal vertical plate with constant mass flux. Ain Shams Engineering Journal 5, 1317-1323.

[10] Mohana Ramana R Girish Kumar J.(2015) Viscous dissipation effects on MHD flow past a parabolic started plate with variable temperature and mass diffusion International journal $O f$ Scientific \& Engineering Research, Vol. 6, Issue 9, pp 57-62.

[11] Jyotsna Rani Pattnaik,Gouranga C. D, Suprava.S (2017) Radiation and mass transfer effects on MHD flow through porous medium past an exponentially accelerated inclined plate with variable temperature. Ain Shams Engineering Journal Vol 8. Issue 7 pp 67-75.

[12] Gaurav Kumar Sharma, Lav Kush Kumar,Navneet Kumar Singh (2016) Unsteady Flow through Porous Media Past on Moving Vertical Plate with Variable Temperature in the Presence of Inclined Magnetic Field. International journal of innovative technology and research (ijtr)Vol. No.4, Issue No.2, pp $2784-2788$. 